\title{
Performance in Later Life
}

\author{
Petra Jansen', Katharina Dahmen-Zimmer', \\ Brigitte M. Kudielka', and Anja Schulz ${ }^{2}$
}

\begin{abstract}
In a randomized controlled trial, we investigated the effects of karate versus a mindfulness-based stress reduction (MBSR) intervention on well-being and cognitive functioning in older adults. Fifty-five adults (52-8I years old) participated in twice-weekly karate versus MBSR sessions or no training for 8 weeks. In pre- and postassessments, subjective well-being, health, cognitive functioning, and chronic stress were measured. Preassessment hair cortisol served as physiological stress marker. The results showed an improvement for the karate group, but not the MBSR and control group, in subjective mental health and anxiety as well as cognitive processing speed. The MBSR group showed by trend as a decrease in stress. No significant correlation between preassessment hair cortisol and postassessment outcomes could be established. But the higher the level of baseline self-reported perceived stress, the higher the increase in depression, anxiety, and chronic stress.
\end{abstract}

\footnotetext{
' Department of Psychology, Education and Sport Science, University of Regensburg, Regensburg, Germany

${ }^{2}$ Clinic for Forensic Psychology and Psychotherapy, Parsberg, Germany
}

\section{Corresponding Author:}

Petra Jansen, Department of Psychology, Education and Sport Science, University of Regensburg, Regensburg 93047, Germany.

Email: petra.jansen@ur.de 
Generally, it can be assumed that karate and MBSR showed only small training effects concerning the assessed emotional and cognitive parameters.

\section{Keywords}

karate, mindfulness, intervention, emotional well-being, cognitive functioning, hair cortisol

Older adults face many biopsychosocial stressors (Kahana, Kahana, \& Lee, 2014). These stressors may include increased job strain due to reduced ability to adjust to time pressure and job demands (Virtanen et al., 2015), declining health and limited mobility (Lee et al., 2012), or alternatively the decline of memory, reasoning, as well as phonemic and semantic fluency after retirement (Singh-Manoux et al., 2012). There is evidence that an accumulation of such stressors may cause depression (Tsai, Chi, \& Wang, 2015). In turn, chronic negative affect has been found to increase cortisol levels as physiological stress markers among older adults (Piazza, Charles, Stawski, \& Almeida, 2013).

Since these late life stressors can have such negative consequences, it appears crucial to identify training methods that teach effective stress regulation to increase well-being and counteract cognitive decline in older adults. To date, some evidence has been accumulated on the role of physical activity and mindfulness-based interventions on emotional well-being and cognitive functioning.

\section{The Role of Physical Activity in Emotional Well-Being and Cognitive Functioning}

There is extensive empirical evidence that physical activity is beneficial for reducing symptoms of depression. Previous meta-analytic reviews yielded moderate to large effects when comparing exercise conditions to waitlist control conditions (Lawlor, 2001). In these meta-analytic reviews (e.g., Craft \& Landers, 1998), the effects of different exercise types (running, running and walking, walking, nonaerobic activity, and other aerobic activity) were comparable. When specifically addressing samples of older adults, similar findings emerged (Strawbridge, Deleger, Roberts, \& Kaplan, 2002). Moreover, evidence shows that regular, not sporadic exercise is associated with improved mood in older adults, including an enhanced feeling of competency and self-efficacy. Mood-improving effects were found for all types of exercise (cardiovascular, resistance training, mixed), but particularly for 
resistance training (Arent, Landers, \& Etnier, 2000), when exercise was practiced fewer than 3 days per week, for more than 45 min or adjusted to participant's individual needs, and with low-to-medium intensity.

Concerning cognitive functioning, Colcombe and Kramer (2003) analyzed, in their meta-analytic review, if physical activity enhances cognitive functions of healthy older adults. The authors differentiated between a cardiovascular only training and a combined strength and aerobic training. They found the highest benefit of physical activity for executive functions, including cognitive control and speed, however, spatial processes also improved. Older groups showed larger effects than younger groups. Furthermore, benefits were more pronounced after participation in a combined strength and aerobic training than in aerobic training alone. Yet, in a later meta-analysis of 11 aerobic intervention studies with older adults (55 years or older), Angevaren, Aufdemkampe, Verhaar, Aleman, and Vanhees (2008) highlighted that there appeared to be large differences concerning the kind of cognitive functioning that showed improvement following physical exercise and that many training effects remained nonsignificant. These authors found beneficial effects on motor function, cognitive speed, delayed memory functions, and auditory and visual attention. Moreover, a recent systematic review did not find an effect of physical exercise on cognitive functioning (Young, Angevaren, Rusted, \& Tabet, 2015). The incongruent findings reflect different research strategies, Colcombe and Kramer included quasiexperimental studies whereas Angevaren et al. (2008) and Young, Angevaren, Rusted, and Tabet (2015) exclusively focused on randomized controlled designs.

So far, much research focused on cardiovascular fitness and aerobic exercise. One specific kind of physical exercise that may be in particular beneficial for well-being and cognitive functioning is karate, a form of martial arts, practiced for self-defense and to improve health. The physical component of karate entails moving forward and backward, while simultaneously performing arm- and/or leg movement techniques. Arm techniques are divided into a group of receiving techniques, executed to defend oneself against attacks, and a group of techniques to attack with fists, open hands, or fingers (punch, strike). Leg techniques (kicks) are used in forward, sideways, and backward directions. The lower abdomen (hara) is meant to ensure stability through motion. Karate involves the whole body and combines motor activity with the cognitive demand of learning long sequences of motor movements (i.e., kata). Surprisingly, studies on the potential benefits of karate training on emotional well-being and cognitive functioning are extremely scarce. Muiños and Ballesteros (2015) showed that older karate trainees had increased 
dynamic visual acuity, a marker of perceptual processing speed, compared to sedentary controls. In a study with older adults (67-93 years), the karate group indeed improved in emotional well-being compared to a control group but did not improve in cognitive functioning (Jansen \& Dahmen-Zimmer, 2012).

\section{The Role of Mindfulness in Emotional Well-Being and Cognitive Functioning}

Mindfulness is described as the ability to be in the present moment without judging the situation (Kabat-Zinn, 1990). Mindfulness-based stress reduction (MBSR) is a program originally designed to reach this state. The individual learns how to observe experiences, instead of being completely immersed. Mindfulness training can reduce symptoms of stress and negative feelings and also disturbances in mood (Brown \& Ryan, 2003). In a preliminary study with older adults who showed increased worrying and some cognitive dysfunctions, Lenze et al. (2014) showed that worries decreased after an MBSR training of either 8 or 12 weeks. De Frias and Whyne (2015) showed that adults with higher levels of trait mindfulness showed a decreased negative effect of life stress on mental health.

The number of studies, which investigate the effect of mindfulness-based interventions on age-related cognitive decline, is rather small. Moreover, studies are difficult to compare due to the use of a different meditation techniques. However, there are some preliminary reports on positive effects on attention, memory, executive function, processing speed, and general cognition (Gard, Hölzel, \& Lazar, 2014). More specifically, Moynihan et al. (2013) showed that MBSR training with older adults (age 65 or older) yielded small but significant changes in executive functions compared to a waiting control group. In the study of Lenze et al. (2014), the MBSR training group showed large improvements in worry severity as well as in memory as measured by paragraph learning and recall after a delay. Furthermore, there is evidence for a relationship between trait mindfulness and executive functioning, in particular set shifting, which is mediated by perceived stress in older people (Fiocco \& Mallya, 2015).

In contrast to these positive results, there were also studies showing no effect of mindfulness-based training on cognitive functions. For example, Anderson, Lau, Segal, and Bishop (2007) could not find a benefit for attentional control after an 8-week MBSR program. Also, Lykins, Baer, and Gottlob (2012) did not find an advantage of long-term mindfulness meditators over demographically matched nonmeditators in attentional control. Both studies were comprised of young adults (give age range). In the study of Mallya and Fiocco (2016), older adults (age range) served as participants; however, again no increase in cognitive functions could be found after MBSR training. 


\section{Differential Effects of Karate and MBSR on Emotional Well-Being and Cognitive Performance}

The review of the intervention literature suggests that both karate training and MBSR may affect well-being and cognitive functioning in later life. Whereas MBSR focuses on teaching purposeful nonjudgmental awareness and attention, karate combines physical exercise with cognitive training and thus can induce "time out" from negative thoughts and worries (cf. Jansen \& Dahmen-Zimmer, 2012) while additionally including the aspect of physical activity. The present study aims to investigate whether karate training and MBSR differentially contribute to increased emotional well-being and cognitive functioning in older adults. For cognitive functioning, we chose to investigate mental rotation, working memory, and cognitive processing speed, because those variables have been shown to relate to both physical activity (e.g., Pietsch \& Jansen, 2012) and mindfulness-based interventions (Gard et al., 2014). To capture the full range of emotional well-being, we focused on both the reduction of depression and the enhancement of positive emotions. In addition, accounting for the relevance of normative biopsychosocial stressors in later adult life, stress was measured on a subjective as well as physiological level, and the impact of stress on the extent of change was assessed.

\section{Method}

\section{Participants}

Participants were recruited via advertisements in the local newspaper. Exclusion criteria included previous heart attack, heart surgery, neurological diseases (e.g., stroke, Morbus Parkinson), severe orthopedic illnesses, dementia, or psychiatric disorders. Initially, 102 participants were interested in the study. Participants were randomly assigned to one of the three groups: the karate training group, the MBSR training group, or the no training control group. A total of 36 volunteers left the study prior to preassessment because they refused to attend the classes they were assigned to, resulting in 66 participants (karate: $n=25$, MBSR: $n=19$, and control group: $n=22$ ) who completed preassessment and two thirds of the training (66\% of total training sessions). After inclusion, 11 participants dropped out during the study without giving any reason (karate: $n=2$, MBSR: $n=4$, and control group: $n=5)$. Thus, the final sample of the study comprised 55 participants aged 52-81 years old $(M=63.5 ; S D=5.7)$, of whom, 21 were male $(38.2 \%)$ and 33 were female $(60.0 \%)$. One person did not provide information on sex $(1.8 \%)$. 


\section{Procedure}

Before participation, all volunteers received an information letter and written consent was acquired. All demographic and psychological measures, as well as the hair samples, were collected at baseline within 1 week prior to the first training session (Time 1). The tests were individually administered at the same time during the day for the pre- and posttest in a quiet room of the university and each session lasted around $75 \mathrm{~min}$. First, questionnaires were completed followed by the cognitive tests. During testing, participants were not allowed to think aloud. Between each test, there was a short break and refreshments were provided. At the end of the session, hair samples were collected to investigate hair cortisol concentration (HCC). Subsequently, both training groups received 15 training sessions over a period of 8 weeks, that is, training was performed twice a week (with the exception of 1 week in which training was only conducted once due to a holiday). During this time period, the control participants did not receive any intervention. The trainings were conducted in morning group sessions at the Sports Center of the University. Psychological measures were reassessed in the morning for all three groups after 8 weeks (Time 2). For the karate and the MBSR group, the postassessment immediately followed the last training session. The control group was reassessed in the same week. The study was conducted according to the guidelines regarding human experimentation in the declaration of Helsinki and approved by the ethical board of the German Society of Psychology.

\section{Interventions}

The karate training group performed Shotokan karate (karate-Do) according to the guidelines of the Deutscher Karate Verband (DKV, German Karate Federation). Karate-Do involves Kihon, Kumite, and Kata. Kihon involves performing specific movements with legs or arms, and combinations of both, kumite consists of training with a partner, kata is the execution of a variety of tactical fighting exercises (Kanazawa, 2006). The participants learned to carry out attacking and defending techniques together with the partner. Emphasis was put on cooperative training to help both partners to benefit from the training. Participants learned simultaneous movements of legs and arms and exercised partner trainings. With respect to Kata, participants learned the "Heian Shodan." The training was conducted by an experienced senior male karate trainer. He has a license from the DKV $(>20$ years of training experience, including older participants). 
The mindfulness-based intervention was based on the MBSR by KabatZinn (2003). MBSR focuses on teaching mindful awareness that is characterized by a nonevaluative, continuous moment-to-moment awareness of one's experience. The MBSR is composed of didactic and practice elements. It incorporates sitting and walking meditation, body scan exercises, and mindfulness communication. In all these elements, training focuses on the perception of and attention to one's own body, mental states, and emotions. The training was conducted by a professional experienced male teacher for MBSR. There were no specific partner tasks. MBSR and karate training lessons each lasted around $60 \mathrm{~min}$.

\section{Materials}

Demographic data. Single-item questions assessed participants' age, sex, and physical activity (yes/no). Participants reported somatic illnesses in open text fields.

Training adherence. The trainers maintained a list of the weekly training participation.

\section{Measures of emotional well-being}

Subjective well-being. A Multidimensional Mood Questionnaire ("Mehrdimensionaler Befindlichkeitsfragebogen" [MDBF]; Steyer, Schwenkmezger, Notz, \& Eid, 1997) was used to assess subjective well-being. The questionnaire includes 24 items with a 5-point rating scale ranging from 1 (not at all) to 5 (very much) that are grouped into the subscales Mood (elevated vs. depressed mood), Fatigue (wakefulness vs. sleepiness), and Alertness (calmness vs. restlessness). Cronbach's $\alpha$ was reported with .86 to .94 for the subscales (Steyer et al., 1997).

Anxiety and depression. The Hospital Anxiety and Depression Scale (HADS; Zigmond \& Snaith, 1983; German version by Herrmann-Lingen, Buss, \& Snaith, 1995) is a 14-item screening tool to assess the state of anxiety (HADS-anxiety) and depression (HADS-depression) with 7 items each. Answers are given on a 4-point Likert-type scale $(0=$ mostly to $3=n o t$ at all for positive and inversely coded scales for negative items), so that higher scores indicate higher symptom severity (possible range 0-21). Cronbach's $\alpha$ was .73 for HADS-anxiety and .78 for HADS-depression (Herrmann-Lingen et al., 1995). HADS values $\leq 7$ are inconspicuous, values $>11$ are pathological. 
Optimism and pessimism. The Life Orientation Test-Revised (LOT-R; Scheier, Carver, \& Bridges, 1994; German version by Glaesmer, Hoyer, Klotsche, \& Herzberg, 2008) is a self-report instrument that measures individual differences in generalized optimism versus pessimism with 10 items (three positive optimism, three negative pessimism items, and four filler items). The response format is a 5-point Likert-type scale ranging from 0 (strongly disagree) to 4 (strongly agree), with higher sum values indicating stronger optimism or pessimism, respectively (possible range 0-12). Cronbach's $\alpha$ was .69 for optimism and .59 for pessimism (Scheier et al., 1994).

Subjective health. The 12-Item Short-Form Health Survey (SF-12; Ware, Kosinski, \& Keller, 1996) is a standard tool to assess health-related quality of life. It measures subjective physical and mental health by 12 items with higher scores indicating better health-related status. The physical and mental summary health score can be calculated following the scoring algorithm outlined in the manual, rendering scores with a mean of $50( \pm 10 S D)$. The scale was empirically shown to be valid in association with other physical and mental health measures (Ware et al., 1996). We applied the German version as introduced by Bullinger and Kirchberger (1998).

\section{Psychometric measures of cognitive functioning}

Mental rotation. The mental rotation performance was measured using the mental rotation (Peter et al., 1995) test. It is comprised of 24-cube figures created by Shepard and Metzler (1971), which are separated into two sets of 12 items each. The items are printed on four A4 sheets of paper, equaling 6 items per sheet. Each item represents a row of five stimuli, one target stimulus on the left side and four sample stimuli on the right. Participants are instructed to mark the two sample stimuli that are identical but rotated versions of the target stimulus. Participants are given a 3-min time limit for the first 12 items, followed by a 4-min break and another $3 \mathrm{~min}$ for the second set of 12 items.

Cognitive processing speed. The number connection test ("Zahlenverbindungstest" [ZVT]; Oswald \& Roth, 1987) was used to assess cognitive processing speed. The test consists of four sheets of paper, each with a matrix of the numbers 1-90 presented in a scrambled order. Participants have to connect the numbers in ascending order as fast and correctly as possible. The ZVT has satisfactory convergent validity evident in its high correlations with diverse standard IQ tests ( $r=.60$ to .80 ; Vernon, 1993). In the present study, we calculated $t$-values from the participants' mean response times across all four trials, with higher scores indicating better cognitive processing speed. 
Inhibition. Inhibition was measured using the stroop color-word interference test (Bäumler, 1985). This version of the Stroop test consists of three tasks. The participants first read color words (i.e., blue, green, red, and yellow) printed in black ink (reading score), then they name the color of small square patches (nomination score). Finally, they are asked to name the color of the ink in which the color words are printed. Thereby, the color of the ink is incongruent with the semantic meaning of the word (interference score). In the interference task, the automatic response (reading) has to be inhibited. For each task, the participant is shown a A4 sheet of paper with 72 items in three columns. Each task is repeated three times with new items. We calculated $t$-values of the interference score with higher scores indicating better inhibitory performance.

Working memory. The verbal working memory was assessed using the digit span task (German version by Petermann, 2012) in which participants listen to series of digits (e.g., 5-8-2, 3-2-7-9) which they have to repeat either in the same (forward) or reversed (backward) order. The test includes 28 items, and the number of digits per series increases by one until a participant fails to remember 2 items of the same length. One point is given for each item a participant recounts correctly, resulting in total sum scores ranging from 0 to 28 , with higher scores indicating better working memory performance. The digit span task was able to differentiate nonclinical and clinical samples with disorders related to memory deficits (Conklin, Curtis, Katsanis, \& Iacono, 2000), thus showing adequate validity.

\section{Stress assessment}

Perceived stress. Perceived stress was measured using the Trier Inventory for Chronic Stress (TICS; Schulz \& Schlotz, 1999; Schulz, Schlotz, \& Becker, 2004). The TICS comprises 57 items in nine subscales (work overload, social overload, pressure to perform, work discontent, excessive demand at work, lack of social recognition, social tensions, social isolation, and chronic worrying). The TICS also includes a 12-Item Screening Scale as a global measure for chronic stress. Subjects rate how often specific stressful situations were experienced during the last 3 months on a 5-point Likert-type scale $(0=$ never to $4=$ very often $)$ with higher values indicating more stress. The scale is adequately reliable (Cronbach's $\alpha$ ranging from .84 to .9; Schulz et al., 2004).

Hair cortisol concentration. We assessed HCC as physiological indicator of chronic stress before the start of the trainings in the two training groups. HCC is a new, intraindividually stable measure of chronic HPA axis activity that 
can provide a retrospective calendar of accumulated cortisol exposure (Meyer \& Novak, 2012; Russell, Koren, Rieder, \& Van Uum, 2012; Stalder $\&$ Kirschbaum, 2012). It has been shown that the amount of hair cortisol relates to increased chronic stress in different contexts (Staufenbiel, Penninx, Spijker, Elzinga, \& van Rossum, 2013). We collected hair samples of 10-20 mg as described in Kirschbaum, Tietze, Skoluda, and Dettenborn (2009) by cutting two hair strands (minimum 1-cm segments) from two sites in a posterior vertex position as close as possible to the scalp. A commercially available immunoassay with chemiluminescence detection (IBL, Hamburg, Germany) was used to determine cortisol concentration. The intraassay and interassay coefficients of variation were below $10 \%$. The samples were analyzed in the Laboratory of Prof. Kirschbaum (Dresden, Germany).

\section{Statistical Analysis}

Descriptive sample characteristics and training adherence were analyzed with $\chi^{2}$ tests for categorical variables and analyses of variance (ANOVAs) were performed for metric dependent variables. Thus, in order to investigate the effect of karate or MBSR training versus the control group on emotional well-being and cognitive functioning, we conducted $3 \times 2$ repeated measures ANOVAs with group (between-group factor) and time (within-group factor) as independent variables. Significance levels were set at $\alpha=.05$. Post hoc analyses of within-group changes were conducted using dependent Bonferroni-corrected $t$-tests. Bivariate Pearson correlation analyses were used to explore the association between treatment adherence and change (i.e., pre-post difference scores) in the dependent variables. Moreover, to address the association between changes over the training period and chronic stress level, we conducted bivariate Pearson correlations of self-reported chronic stress (i.e., sum score of TICS Screening Scale) and physiological stress (i.e., accumulated cortisol in hair) at preassessment with the pre-post difference scores of all dependent variables.

\section{Results}

\section{Descriptive Statistics and Training Adherence}

Sample characteristics are shown in Table 1. The participant groups did not differ significantly concerning sex, age, physical activity, or somatic illnesses.

As mentioned above, 11 participants dropped out during the course of the study; four participants who had begun MBSR training (21.1\%), two 
Table I. Demographic Characteristics of the Study Groups.

\begin{tabular}{|c|c|c|c|c|c|}
\hline & $\begin{array}{c}\text { Karate } \\
(n=23) \\
\%(n)\end{array}$ & $\begin{array}{c}\text { MBSR } \\
(n=\mid 4) \\
\%(n)\end{array}$ & $\begin{array}{c}\text { Control } \\
(n=17) \\
\%(n)\end{array}$ & $\chi_{(\mathrm{df}=2)}^{2}$ & $P$ \\
\hline Sex (female) & $73.9(17)$ & 57.1 (8) & 47.1 (8) & 3.09 & .213 \\
\hline Age $(M, S D)$ & $62.57(4.19)$ & $63.29(8.53)$ & $65.24(4.66)$ & $1.09^{\mathrm{a}}$ & .346 \\
\hline Physical activity (yes) & $73.9(17)$ & $71.4(10)$ & $70.6(12)$ & 0.06 & .971 \\
\hline Somatic illness (yes) & $45.5(10)$ & $69.2(9)$ & $31.3(5)$ & 4.19 & .123 \\
\hline Hair cortisol (pg/mg) & II.I4 (7.75) & $15.56(22.01)$ & $12.74(14.43)$ & 1.63 & .333 \\
\hline
\end{tabular}

Note. $N=55$.

${ }^{\mathrm{a}} \mathrm{F}$-statistic of univariate analysis of variance.

participants who had begun karate training (8\%), and five participants $(22.7 \%)$ of the control group. These dropouts did not provide any reason for ending their participation. Subjects who dropped out ( 8 females, 3 males) were significantly older $(M=69.42, S D=8.62)$ than the subjects who stayed in the study $(M=63.59, S D=5.74), F(1,64)=8.30, p<.01, \eta^{2}=.115$. The two groups differed significantly in only one of the nine dependent measurements, with the dropped-out subjects showing a reduced processing speed, $F(1,64)=9.35, p<.01, \eta^{2}=.127$, during pretesting.

In the remaining sample $(n=55)$, the mean number of completed training sessions in the intervention groups was $13.0(S D=1.75)$ with a range of $8-15$ sessions. The karate group $(M=13.26 ; S D=1.48)$ and the MBSR group $(M=12.60 ; S D=2.10)$ did not differ significantly concerning the number of completed training sessions, $F(1,36)=1.30, p=.262$. The number of completed training sessions was not significantly correlated with the extent of change in any dependent measure of emotional well-being or cognitive performance.

\section{Emotional Well-Being}

Subsequently, we investigated the impact of training on subjective wellbeing in terms of mood, fatigue, and agitation (MDBF), symptoms of anxiety and depression (HADS), optimism and pessimism (LOT-R), as well as subjective physical and mental health (SF-12; Table 2).

The repeated measure analysis yielded a significant main effect of group for self-reported symptoms of fatigue $\left(p=.012, \eta^{2}=.16\right)$. Bonferronicorrected post hoc analyses revealed that the control group reported less fatigue than the karate group $(p=.010)$, whereas no significant difference 
Table 2. Descriptive Statistics and F-statistics of Repeated Measure Analyses of Variance of Emotional Well-Being and Subjective Health as a Function of Group and Time $(N=55)$.

\begin{tabular}{|c|c|c|c|c|c|c|}
\hline $\begin{array}{l}\text { Dependent } \\
\text { Variable }\end{array}$ & Group & $\begin{array}{c}\text { Pre } \\
M(S D)\end{array}$ & $\begin{array}{c}\text { Post } \\
M(S D)\end{array}$ & $\begin{array}{l}\text { Time } \\
F_{(d f=1)}\end{array}$ & $\begin{array}{l}\text { Group } \\
F_{(d f=2)}\end{array}$ & $\begin{array}{c}\text { Time } \times \\
\text { Group } \\
F_{(d f=2)}\end{array}$ \\
\hline \multirow[t]{3}{*}{ MDBF mood } & Karate & II.I8 (1.94) & II.I4 (I.36) & 0.10 & 3.01 & 1.08 \\
\hline & MBSR & II.I3 (2.23) & II.67 (I.29) & & & \\
\hline & Control & $10.82(I .5 I)$ & $10.00(2.21)$ & & & \\
\hline \multirow[t]{3}{*}{ MDBF fatigue } & Karate & $12.00(2.27)$ & $\mathrm{I} 1.22$ (1.77) & 2.79 & $4.87^{*}$ & 0.71 \\
\hline & MBSR & 10.59 (1.23) & II. 20 (0.86) & & & \\
\hline & Control & 10.59 (I.23) & 10.00 (I.58) & & & \\
\hline \multirow[t]{3}{*}{ MDBF agitation } & Karate & $10.86(1.46)$ & I I.27 (I.58) & 2.25 & 0.62 & 0.24 \\
\hline & MBSR & $10.47(2.70)$ & II.27 (I.16) & & & \\
\hline & Control & $10.4 \mid(2.27)$ & $10.65(2.09)$ & & & \\
\hline \multirow[t]{3}{*}{ HADS-anxiety } & Karate & $7.47(4.15)$ & $5.82(3.88)$ & 2.47 & 0.39 & $4.05^{*}$ \\
\hline & MBSR & $7.26(3.78)$ & $6.73(3.32)$ & & & \\
\hline & Control & $5.64(3.55)$ & $6.23(3.13)$ & & & \\
\hline \multirow[t]{3}{*}{ HADS-depression } & Karate & $4.68(3.82)$ & $3.69(3.26)$ & 2.39 & 0.32 & 1.49 \\
\hline & MBSR & $5.40(4.23)$ & $4.53(3.44)$ & & & \\
\hline & Control & $4.58(1.76)$ & $4.88(2.62)$ & & & \\
\hline \multirow[t]{3}{*}{ LOT-R optimism } & Karate & $8.78(2.88)$ & $9.22(2.43)$ & 0.56 & 0.03 & 0.29 \\
\hline & MBSR & $8.80(2.37)$ & 8.93 (1.83) & & & \\
\hline & Control & $9.06(2.46)$ & $9.06(2.28)$ & & & \\
\hline \multirow[t]{3}{*}{ LOT-R pessimism } & Karate & $5.09(2.79)$ & $4.13(2.72)$ & 0.88 & 0.18 & 1.42 \\
\hline & MBSR & $4.73(2.58)$ & $4.40(2.82)$ & & & \\
\hline & Control & $4.00(2.18)$ & $4.35(2.74)$ & & & \\
\hline \multirow[t]{3}{*}{ SF-12 physical } & Karate & 46.97 (8.27) & $45.28(9.55)$ & 1.62 & 1.01 & 0.45 \\
\hline & MBSR & 50.31 (6.69) & 49.76 (6.77) & & & \\
\hline & Control & $48.65(8.54)$ & $48.32(10.50)$ & & & \\
\hline \multirow[t]{3}{*}{ SF-I 2 mental } & Karate & 47.47 (12.97) & $54.70(5.85)$ & $5.20 *$ & 1.01 & $3.19 *$ \\
\hline & MBSR & 47.11 (10.82) & $50.66(7.00)$ & & & \\
\hline & Control & $53.19(6.05)$ & $52.10(6.74)$ & & & \\
\hline
\end{tabular}

Note. MDBF $=$ Multidimensionaler Befindlichkeitsfragebogen (Multidimensional Mood Questionnaire); $M B S R=$ mindfulness-based stress reduction; HADS $=$ Hospital Anxiety and Depression Scale; LOT-R = Life Orientation Test-Revised. SF-I2 = Short Form of the Health Survey12 Item Version. TICS $=$ Trier Inventory for Chronic Stress.

$*_{p}<.05$.

emerged between the control group and the MBSR group $(p=.175)$ or between the karate and the MBSR group $(p>.999)$. As shown in Table 2, there was no significant main effect of group on any other measure of wellbeing or subjective health. 
There was a significant main effect of time concerning the mental summary score of the SF-12 $\left(p=.027, \eta^{2}=.10\right)$, indicating that perceived mental health improved significantly from preassessment to postassessment. Yet, there was also a significant interaction effect of Group $\times$ Time $(p=.05$, $\eta^{2}=.12$ ). Post hoc analyses revealed that this improvement was only significant within the karate group $(p=.011)$, but not significant in the MBSR group $(p=.242)$, nor in the control group $(p=.320)$. Furthermore, a significant Group $\times$ Time interaction emerged for HADS-anxiety $(p=.023$, $\left.\eta^{2}=.135\right)$. Post hoc analyses revealed that this improvement was only significant within the karate group $(p=.008)$, but not in the MBSR group $(p=.465)$, nor in the control group $(p=.181)$.

As shown in Table 2, no other significant main effects of time or interaction effects of training Group $\times$ Time emerged in relation to measures of well-being or subjective health.

\section{Cognitive Performance}

Results regarding cognitive performance are summarized in Table 3.

There was a main effect of group for mental rotation performance (mental rotation; $p=.012, \eta^{2}=.16$ ), cognitive processing speed (ZVT; $p=.007$, $\eta^{2}=.18$ ), as well as working memory performance (digit span; $p<.001$, $\eta^{2}=.29$ ). Bonferroni-corrected post hoc analyses of these group effects revealed a better mental rotation performance in the control group compared to the karate group $(p=.020)$ and the MBSR group $(p=.042)$. The control group also outperformed the MBSR group in the cognitive processing speed $(p=.006)$, but there was no significant difference between the control group and the karate group $(p=.106)$. Concerning working memory performance in the digit span task, the control group remembered significantly more numbers than both the karate group $(p=.001)$ and the MBSR group $(p<.001)$. Post hoc analyses showed that the karate group and the MBSR group did not differ significantly on any of these measures (mental rotation: $p>$.999; ZVT: $p=.492$; digit span: $p>$.999). As shown in Table 3, there was no main effect of training group concerning the inhibitory performance in the Stroop task.

The analyses yielded significant main effects of time for all indicators of cognitive function. As shown in Table 3, participants' performance improved significantly from preassessment to postassessment concerning the mental rotation $\left(p<.001, \eta^{2}=.23\right)$, inhibition $\left(p<.001, \eta^{2}=.24\right)$, and cognitive processing speed $\left(p<.001, \eta^{2}=.35\right)$. In contrast, the working memory performance, that is, the number of remembered digits 


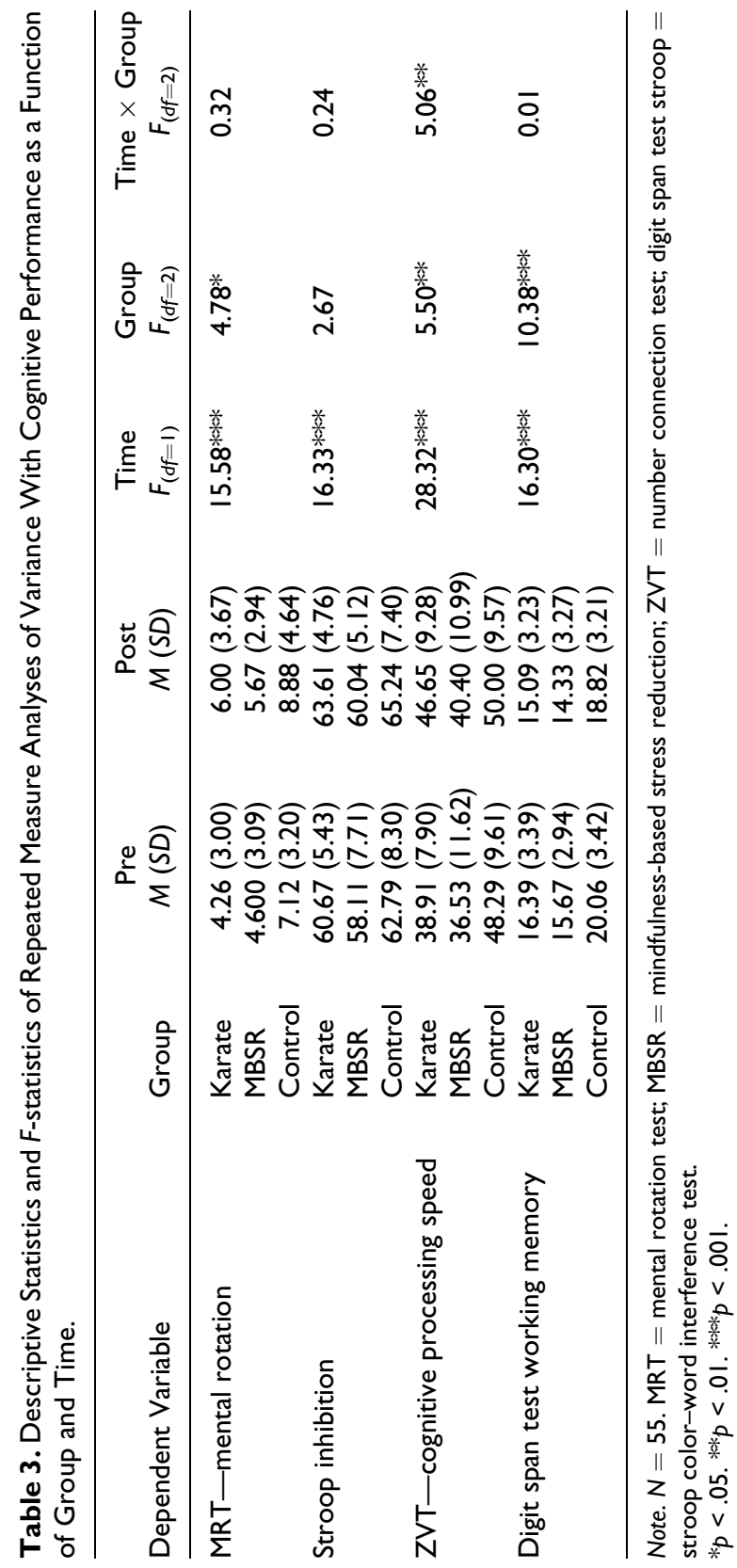


in the digit span task, decreased significantly from pre- to postassessment $\left(p<.001, \eta^{2}=.24\right)$.

There was no significant interaction effect between group and time for mental rotation $\left(p=.726, \eta^{2}=.01\right)$, inhibition $\left(p=.789, \eta^{2}=.01\right)$, or working memory performance $\left(p<.992, \eta^{2}<.01\right)$. However, a significant interaction effect of group and time emerged for the information processing speed in the ZVT $\left(p=.010, \eta^{2}=.16\right)$. Post hoc analyses revealed that the cognitive processing speed improved significantly only in the karate group $(p<.001)$, whereas no improvement emerged in the MBSR group, $(p=.100)$ or the control group $(p=.183)$.

\section{Stress Variables and Correlation between Baseline Stress-Level and Pre-post changes}

Concerning the level of self-reported chronic stress, the repeated measure ANOVA yielded no significant main effects of group or time on any of the TICS subscales (see Table 4). Similarly, no significant interaction effects between group and time emerged with the exception of the subscale "lack of social support" (TICS LSR; $p=.044, \eta^{2}=.12$ ). While there was no difference between the training groups at preassessment, $F(2,54)=1.04$, $p=.362, \eta^{2}=.04$, a difference in lack of social support emerged at postassessment, $F(2,54)=4.37, p=.018, \eta^{2}=.15$. Bonferroni-corrected post hoc analyses revealed that the MBSR group reported significantly less lack of social support than the control group $(p<.021)$. No difference emerged between the MBSR and the karate group $(p>.999)$ or the karate group and the controls $(p=.063)$. Concerning stress from "lack of social support," the karate group did not improve significantly from pre- to postassessment $(p=.576)$. In the MBSR group, there was a trend toward decreased stress $(p=.09)$, while there was a trend toward an increase at postassessment in the control group $(p=.085)$.

As shown in Table 4, the two training groups did not significantly differ at preassessment with regard to HCC.

Subsequently, we correlated self-reported (TICS Screening Scale) and physiological (hair cortisol) stress indicators at preassessment with the amount of change in the dependent variables from pre- to postassessment (i.e., difference scores of postminus preassessment values; see Table 5). Based on the respective variable's coding, improvements are evident in positive or negative difference scores (positive difference scores: wellbeing in the MDBF, optimism, physical and mental subjective health, cognitive processing speed, inhibitory control, and working memory; negative 
Table 4. Descriptive Statistics and F-statistics of Repeated Measure Analyses of Variance of Psychological and Physiological Stress as a Function of Group and Time.

\begin{tabular}{|c|c|c|c|c|c|c|}
\hline Stress Indicator & Group & $\begin{array}{c}\text { Pre } \\
M(S D)\end{array}$ & $\begin{array}{c}\text { Post } \\
M(S D)\end{array}$ & $\begin{array}{c}\text { Time } \\
F_{(d f=1)}\end{array}$ & $\begin{array}{l}\text { Group } \\
F_{(d f=2)}\end{array}$ & $\begin{array}{c}\text { Time } \times \\
\text { Group } \\
F_{(d f=2)}\end{array}$ \\
\hline \multirow{2}{*}{$\begin{array}{l}\text { Hair cortisol } \\
\quad(\mathrm{pg} / \mathrm{mg})\end{array}$} & Karate & II.I5 (7.75) & - & - & 0.60 & - \\
\hline & $\begin{array}{l}\text { MBSR } \\
\text { Control }\end{array}$ & $\begin{array}{c}|4.9|(2 \mid .29) \\
-\end{array}$ & - & - & & \\
\hline \multirow[t]{3}{*}{ TICS screening } & Karate & $8.09(4.5 I)$ & $7.47(3.87)$ & 0.42 & 0.03 & 1.52 \\
\hline & MBSR & $7.56(3.82)$ & $7.70(3.77)$ & & & \\
\hline & Control & $7.34(2.09)$ & $7.67(2.31)$ & & & \\
\hline \multirow[t]{3}{*}{ TICS WO } & Karate & $9.59(7.04)$ & $9.77(6.29)$ & 0.08 & 0.21 & 0.01 \\
\hline & MBSR & $10.13(6.90)$ & $10.20(6.95)$ & & & \\
\hline & Control & $8.76(5.07)$ & $8.88(4.23)$ & & & \\
\hline \multirow[t]{3}{*}{ TICS SO } & Karate & $9.95(5.64)$ & 9.41 (5.39) & 1.06 & 0.58 & 2.07 \\
\hline & MBSR & $9.00(5.78)$ & $10.93(5.15)$ & & & \\
\hline & Control & $8.23(4.16)$ & $8.41(3.96)$ & & & \\
\hline \multirow[t]{3}{*}{ TICS PP } & Karate & II.50 (8.28) & $10.86(7.40)$ & 0.04 & 0.36 & 0.61 \\
\hline & MBSR & $12.80(7.90)$ & I 3.33 (7.85) & & & \\
\hline & Control & $12.53(6.98)$ & $12.94(6.38)$ & & & \\
\hline \multirow[t]{3}{*}{ TICS WD } & Karate & II.43 (6.73) & $9.76(5.82)$ & 1.53 & 1.46 & 1.70 \\
\hline & MBSR & 7.86 (4.59) & $7.86(3.30)$ & & & \\
\hline & Control & $9.06(3.42)$ & $9.09(3.93)$ & & & \\
\hline \multirow[t]{3}{*}{ TICS EDW } & Karate & $7.10(5.66)$ & $6.56(4.69)$ & 0.13 & 0.05 & 1.92 \\
\hline & MBSR & $6.29(3.73)$ & $6.19(4.35)$ & & & \\
\hline & Control & $6.12(1.36)$ & $6.57(1.66)$ & & & \\
\hline \multirow[t]{3}{*}{ TICS LSR } & Karate & $3.32(3.40)$ & $3.14(2.95)$ & 0.11 & 2.60 & $3.31 *$ \\
\hline & MBSR & $3.07(1.94)$ & $2.40(1.55)$ & & & \\
\hline & Control & $4.29(1.79)$ & $4.94(2.66)$ & & & \\
\hline \multirow[t]{3}{*}{ TICS ST } & Karate & $6.50(4.53)$ & $6.36(4.59)$ & 0.19 & 0.04 & 0.54 \\
\hline & MBSR & $6.20(3.30)$ & $6.13(4.4 I)$ & & & \\
\hline & Control & $5.76(3.35)$ & 6.41 (3.89) & & & \\
\hline \multirow[t]{3}{*}{ TICS SI } & Karate & $6.45(4.70)$ & $5.55(3.62)$ & 1.30 & 0.33 & 1.36 \\
\hline & MBSR & $7.40(4.64)$ & $6.67(4.34)$ & & & \\
\hline & Control & $6.65(3.48)$ & 7.06 (5.39) & & & \\
\hline \multirow[t]{3}{*}{ TICS CW } & Karate & $7.14(4.35)$ & $6.59(3.80)$ & 0.07 & 1.92 & $0.7 \mathrm{I}$ \\
\hline & MBSR & $6.07(3.37)$ & 6.07 (3.59) & & & \\
\hline & Control & $4.65(2.57)$ & $4.94(2.19)$ & & & \\
\hline
\end{tabular}

Note. TICS = Trier Inventory for Chronic Stress; MBSR = mindfulness-based stress reduction; $\mathrm{WO}=$ work overload; SO $=$ social overload; PP $=$ pressure to perform; WD $=$ work discontent; EDW = excessive demands at work; LSR = lack of social recognition; ST = social tensions; $\mathrm{SI}=$ social isolation; $\mathrm{CW}=$ chronic worrying. $*_{p}<.05$. 
Table 5. Pearson Correlation Coefficients of Hair Cortisol and the TICS Global Score with the Pre-Post Difference Scores in Cognitive Performance and Emotional Well-Being in the Training Groups $(\mathrm{N}=37)$.

\begin{tabular}{llcc}
\hline Category & Difference Score Pre-Post & Hair cortisol, $r$ & TICS, $r$ \\
\hline Positive difference score suggesting improvement & & \\
Cognitive performance & Stroop & .043 & -.169 \\
& ZVT & .222 & -.040 \\
& Digit span & .110 & -.167 \\
Emotional well-being & MDBF mood & .047 & -.193 \\
& MDBF fatigue & .161 & .166 \\
& MDBF agitation & .054 & -.088 \\
\multirow{5}{*}{ Subjective health } & LOT-R optimism & -.002 & $-.400^{*}$ \\
& SF-I2 physical & $.373^{*}$ & $.510^{* * *}$ \\
Negative difference score & SF-I2 mental & -.070 & $-.683^{* * *}$ \\
Cognitive performance & MRT & & \\
Emotional well-being & HADS-anxiety & -.233 & -.022 \\
& HADS-depression & -.161 & $.613^{* * *}$ \\
& LOT-R pessimism & -.247 & $.276^{*}$ \\
Psychological stress & TICS screening & -.320 & -.161 \\
\end{tabular}

Note. MRT = mental rotation test; stroop $=$ stroop color-word interference test; ZVT = number connection test; digit span test MDBF = Multidimensionaler Befindlichkeitsfragebogen (multidimensional mood questionnaire). HADS = Hospital Anxiety and Depression Scale. LOT$\mathrm{R}=$ Life Orientation Test-Revised. SF-12 = Short Form of the Health Survey-12 Item Version. TICS $=$ Trier Inventory for Chronic Stress.

$* p<.05 . * * p<.01 . * * * p<.001$.

difference scores: anxiety and depression, pessimism, perceived stress, and mental rotation). When improvements are reflected by positive differences, a positive correlation suggests that higher stress at baseline leads to stronger improvements. On the other hand, the same implication would be made by a negative correlation in which case improvements are reflected in negative differences.

Table 5 is subdivided in two parts: The upper part contains the variables where positive difference scores indicate improvements, the lower part consists of the negative difference scores which indicate improvement. Table 5 shows that self-reported chronic stress at preassessment (screening sum score) was positively correlated with difference scores for somatic health. This indicates that the higher the level of baseline self-reported perceived stress, the stronger the improvement in somatic health. In contrast, preassessment stress was negatively correlated with changes in psychiatric health and 


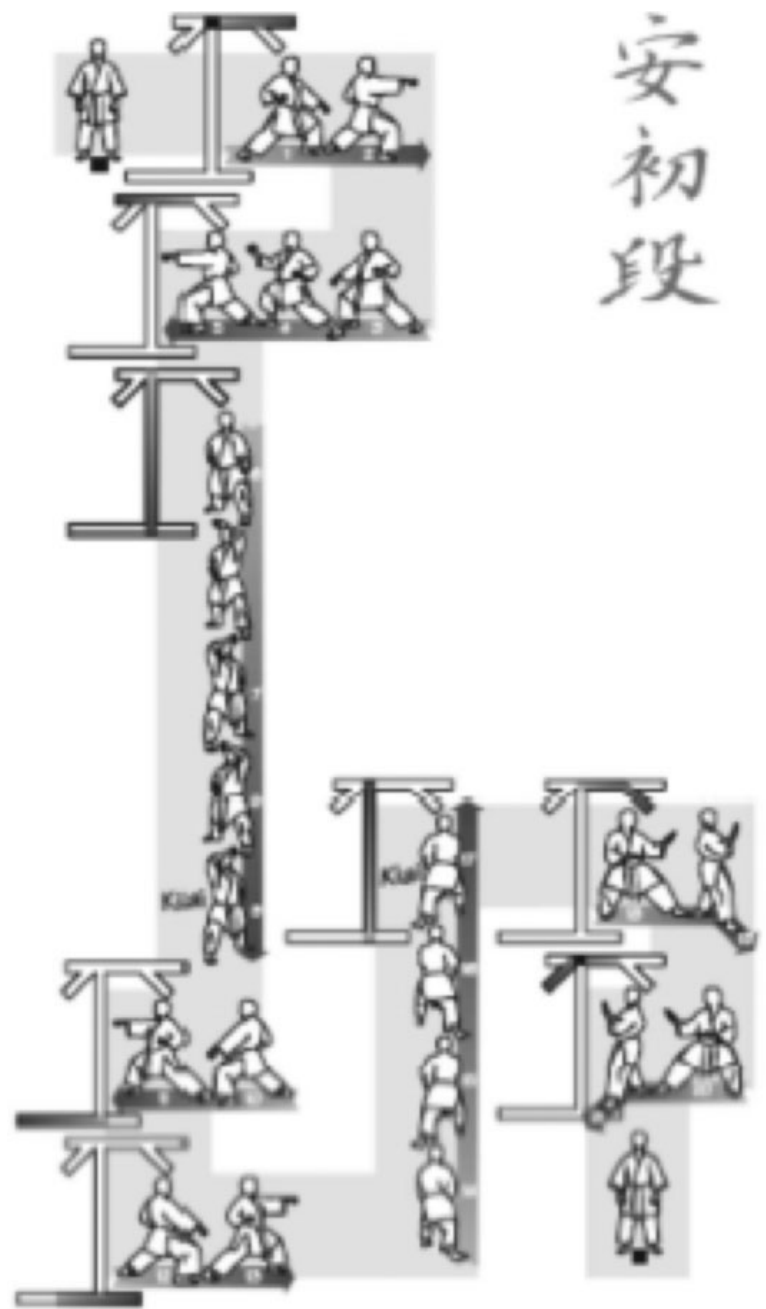

Figure I. Kata "Heian Shodan" (Tartaglia, 2003).

optimism. Both correlations indicate that a higher level of baseline selfreported stress was related to less change or worsening of subjective mental health and optimism. Furthermore, it was shown that an initially higher cortisol level was associated with larger improvement in subjective physical health. 
Finally, self-reported chronic stress at preassessment was positively correlated with difference scores for depression and anxiety as well as chronic stress itself (lower half of Table 5 shows the negative difference scores suggest improvement). Thus, the higher the level of baseline selfreported perceived stress, the higher the increase in depression, anxiety, and chronic stress.

\section{Discussion}

The aim of the present study was to compare the effects of a DKV-karate training and a MBSR training on emotional well-being, subjective health, and cognitive performance. The number of completed training sessions was not associated with the amount of change in well-being and cognitive functioning. Concerning treatment effects, the karate group showed an improvement in cognitive processing speed and subjective mental health and anxiety from pre- to postassessments compared to the MBSR training and control group. The MBSR group showed, by trend, an improvement in perceived stress from lack of social support compared to the control group. No significant effect of the training emerged for mental rotation, inhibition, working memory, or any of the indicators of emotional well-being. Both training groups and the controls improved in mental rotation, inhibition, and cognitive processing speed but worsened in working memory from pre- to postassessment. Higher self-reported stress at preassessment, but not the hair cortisol level, was associated with a stronger increase in depression, anxiety, and chronic stress from pre- to posttraining but less increase in subjective mental health and optimism. However, higher self-reported stress at preassessment was associated with an increase in subjective physical health.

The relatively high treatment adherence, particularly for karate training, may be indicative of a high acceptability among older adults. This is a benefit compared to other medical or psychotherapeutic treatments with typically lower adherence, such as long-term medication (Osterberg \& Blaschke, 2005). Moreover, the lack of association between training adherence and pre-post intervention changes may be a statistical artifact due to the small variance with respect to the number of completed sessions and the use of nonclinical volunteers.

Concerning the changes in cognitive performance only one effect emerged, namely, an improvement of cognitive processing speed in the karate group. This result reflects similar findings of lacking or only small effects in studies that investigated karate and MBSR with regard to executive function (Gard et al., 2014; Moynihan et al., 2013). One explanation may be that these studies, including the present, have sampled healthy participants. 
There is meta-analytical evidence that mindfulness interventions yield larger effects in samples with low base rate functioning (Baer, 2003; Zoogman, Goldberg, Hoyt, \& Miller, 2015). Moreover, the present study did not include a follow-up assessment. Thus, it is not possible to disentangle whether the karate or MBSR training contribute to maintaining, rather than improving, the cognitive performance in comparison to the control group. Another noteworthy fact is the significant effect of time on cognitive functioning, which potentially results from repeated testing with the same instruments within a relatively short period of time ( 8 weeks). Such practice effects may have obliterated training effects, which represent a limitation for the internal validity of this study.

Our study showed a training effect of karate on anxiety and self-rated mental health, which is in line with our former study (Jansen \& DahmenZimmer, 2012). The fact that the MBSR training did not have such an effect is in contrast previous studies (Baer, 2003). However, Mallya and Fiocco (2016) also could not show any positive effects of a mindfulness intervention on emotional well-being in healthy older adults.

The karate training together with a partner was introduced to improve feelings of social support. Instead, a trend toward improvement in perceived stress due to lack of social support was found in the MBSR group but not in the karate group. This may reflect the philosophy of kind and nonjudgmental thinking which is an integral part of the MBSR processes (Hölzel et al., 2011; Kabat-Zinn, 2003). Mindfulness practices may increase the insight into the impermanence of situations and the ability of self-regulation and selfliberation (Kabat-Zinn, 1994), thus empowering individuals and increasing perceived self-efficacy. Moreover, mindfulness practice teaches attending to oneself, others, and different situations with a nonevaluative attitude of patience, acceptance, kindness, and compassion. Consequently, individuals are more forgiving toward others whom they expected support from, thus reducing stress from lack of social support.

The physiological stress indicator (accumulated cortisol in hair) had little association with subsequent treatment change whereas self-reported stress correlated with medium to high effect sizes with the change on several measures. Some of these correlations were unexpected in that high initial stress level were related to stronger increases in anxiety, depression, and perceived chronic stress as well as to decreases in optimism and subjective mental health. The source of this effect remains unclear. One possible explanation - which could be tested in future research - may be that the patients with higher initial stress were under considerable strain with respect to the "success" of the training. Maybe they were eager to feel better after the 
training and somewhat disappointed that changes were subjectively small. A divergence between bio-physiological and psychological measures has been established in other contexts as well. At least with respect to acute stress, it was recently shown that endocrine stress responses lag behind acute psychological responses but that time-lagged correlations lead to a much closer coupling than reported in earlier studies (Schlotz et al., 2008). However, the relationship between self-reported chronic stress and biological measures of chronic stress is still under debate.

The present study has several limitations. It is important to note that the multiple testing may have resulted in type II error inflation. Another limitation is the fairly small sample of healthy volunteers. Furthermore, the short time between pre- and postassessment may have led to practice effects in the cognitive performance measures. Moreover, the postassessment immediately followed the last training session. Thus, the karate and the MBSR group participants may have been tired or at least in a different mood or physical state compared to the baseline assessment. We cannot rule out that these differences in mood or physical state influenced the results. Besides, the three groups differed in the relative number of males and females participating. Interestingly, Colcombe and Kramer (2003) showed that some cognitive beneficial effects were more pronounced if there were more females than males in the training group.

The present study did not include a follow-up assessment which may provide more insight in the maintenance of cognitive functioning and emotional well-being rather than short-term improvements. Unfortunately, educational level was not measured, which should be integrated in future studies. Furthermore, we decided not to give any homework to the MBSR group, as it is usually done (Kabat-Zinn, 2003), because we wanted to keep the amount of practice in the karate and MBSR groups comparable. But surely, this procedure differs from the normal MBSR practice and might contribute to the missing training effects on emotional well-being. Future intervention studies should investigate the effect of different forms of karate and mindfulness based methods could be investigated in more detail, as it has been discussed for Tai Chi (Burschka, Kuhn, Menge, \& Oschmann, 2013).

In conclusion, the present study provided insight into the effects of karate training and a MBSR training on emotional well-being, subjective health, and cognitive functioning. Although the effects were overall small, the findings suggest that karate and MBSR training are acceptable interventions among older adults. Future research should thus address if these trainings are beneficial in the maintenance of emotional and cognitive functioning in older adults. 


\section{Author Contributions}

Petra Jansen and Katharina Dahmen-Zimmer contributed equally to this work.

\section{Declaration of Conflicting Interests}

The author(s) declared no potential conflicts of interest with respect to the research, authorship, and/or publication of this article.

\section{Funding}

The author(s) received no financial support for the research, authorship, and/or publication of this article.

\section{References}

Anderson, N. D., Lau, M. A., Segal, Z. V., \& Bishop, S. R. (2007). Mindfulness-based stress reduction and attentional control. Clinical Psychology and Psychotherapy, 14, 449-463. doi:10.1002/cpp.544

Angevaren, M., Aufdemkampe, G., Verhaar, H. J. J., Aleman, A., \& Vanhees, L. (2008). Physical activity and enhanced fitness to improve cognitive function in older people without known cognitive impairment. The Cochrane Database of Systematic Reviews, 3, 1-56. CD005381. doi:10.1002/14651858.CD005381.pub3

Arent, S., Landers, M., \& Etnier, J. (2000). The effects of exercise on mood in older adults: A meta-analytic review. Journal of Aging and Physical Activity, 8, 407-430.

Baer, R. A. (2003). Mindfulness training as a clinical intervention: A conceptual and empirical review. Clinical Psychology: Science and Practice, 10, 125-143. doi: 10.1093/clipsy/bpg015

Bäumler, G. (1985). Farb-Wort-Interferenztest (FWIT) nach J. R. Stroop (1st ed.). Göttingen, Germany: Hogrefe.

Brown, K. W., \& Ryan, R. M. (2003). The benefits of being present: Mindfulness and its role in psychological well-being. Journal of Personality and Social Psychology, 84, 822-848. doi:10.1037/0022-3514.84.4.822

Bullinger, M., \& Kirchberger, I. (1998). SF-36. Fragebogen zum Gesundheitszustand. Handanweisung. Göttingen, Germany: Hogrefe.

Burschka, J., Kuhn, P., Menge, U., \& Oschmann, P. (2013). Research on Tai Chi as a sport in health care-The challenge of complex interventions. Sportwissenschaft, 43, 181-196. doi:10.1007/s12662-013-0300-1

Colcombe, S., \& Kramer, A. F. (2003). Fitness effects on the cognitive function of older adults: A meta-analytic study. Psychological Science, 14, 125-130. doi:10. 1111/1467-9280.t01-1-01430

Conklin, H. M., Curtis, C. E., Katsanis, J., \& Iacono, W. G. (2000). Verbal working memory impairment in schizophrenia patients and their first-degree relatives: 
Evidence from the digit span task. The American Journal of Psychiatry, 157, 275-277. doi:10.1176/appi.ajp.157.2.275

Craft, L. L., \& Landers, D. M. (1998). The effect of exercise on clinical depression and depression resulting from mental illness: A meta-analysis. Journal of Sport and Exercise Psychology, 20, 339-357.

De Frias, C. M., \& Whyne, E. (2015). Stress on health-related quality of life in older adults: The protective nature of mindfulness. Aging \& Mental Health, 19, 3. doi: 10.1080/13607863.2014.924090

Fiocco, A. J., \& Mallya, S. (2015). The importance of cultivating mindfulness for cognitive and emotional well-being in late life. Journal of Evidence-based Complementary \& Alternative Medicine, 20, 35-40. doi:10.1177/2156587214553940

Gard, T., Hölzel, B. K., \& Lazar, S. W. (2014). The potential effects of meditation on age-related cognitive decline: A systematic review. Annals of the New York Academy of Sciences, 1307, 89-103. doi:10.1111/nyas.12348

Glaesmer, H., Hoyer, J., Klotsche, J., \& Herzberg, P. Y. (2008). Die deutsche Version des Life-Orientation-Tests (LOT-R) zum dispositionellen Optimismus und Pessimismus. Zeitschrift für Gesundheitspsychologie, 16, 26-31. doi:10.1026/09438149.16.1.26

Herrmann-Lingen, C., Buss, U., \& Snaith, R. P. (Eds.). (1995). Hospital Anxiety and Depression Scale: Deutsche Version; HADS-D; ein Fragebogen zur Erfassung von Angst und Depressivität in der somatischen Medizin; Testdokumentation und Handanweisung (1. Aufl.). Bern, Switzerland: Huber.

Hölzel, B. K., Lazar, S. W., Gard, T., Schuman-Olivier, Z., Vago, D. R., \& Ott, U. (2011). How does mindfulness meditation work? Proposing mechanisms of action from a conceptual and neural perspective. Perspectives on Psychological Science: A Journal of the Association for Psychological Science, 6, 537-559. doi:10.1177/ 1745691611419671

Jansen, P., \& Dahmen-Zimmer, K. (2012). Effects of cognitive, motor, and karate training on cognitive functioning and emotional well-being of elderly people. Frontiers in Psychology, 3, 40. doi:10.3389/fpsyg.2012.00040

Kabat-Zinn, J. (1990). Full catastrophe living: The program of the stress reduction clinic at the University of Massachusetts Medical Center. New York, NY: Delta.

Kabat-Zinn, J. (1994). Wherever you go there you are: Mindfulness meditation in everyday life. New York, NY: Hyperion.

Kabat-Zinn, J. (2003). Mindfulness-based interventions in context: Past, present, and future. Clinical Psychology: Science and Practice, 10, 144-156. doi:10.1093/ clipsy/bpg016

Kahana, E., Kahana, B., \& Lee, J. E. (2014). Proactive approaches to successful aging: One clear path through the forest. Gerontology, 60, 466-474. doi:10. 1159/000360222 
Kanazawa, H. (2006). Black belt karate: The intensive course. Bunkyō, Tokyo: Kodansha International.

Kirschbaum, C., Tietze, A., Skoluda, N., \& Dettenborn, L. (2009). Hair as a retrospective calendar of cortisol production-Increased cortisol incorporation into hair in the third trimester of pregnancy. Psychoneuroendocrinology, 34, 32-37. doi:10.1016/j.psyneuen.2008.08.024

Lawlor, D. A. (2001). The effectiveness of exercise as an intervention in the management of depression: Systematic review and meta-regression analysis of randomised controlled trials. British Medical Journal, 322, 763. doi:10.1136/bmj.322. 7289.763

Lee, C.-T., Yeh, C.-J., Lee, M.-C., Lin, H.-S., Chen, V. C.-H., Hsieh, M.-H., . . Lai, T.-J. (2012). Leisure activity, mobility limitation and stress as modifiable risk factors for depressive symptoms in the elderly: Results of a national longitudinal study. Archives of Gerontology and Geriatrics, 54, e221-e229. doi:10.1016/j. archger.2011.06.014

Lenze, E. J., Hickman, S., Hershey, T., Wendleton, L., Ly, K., Dixon, D., . . W Wetherell, J. L. (2014). Mindfulness-based stress reduction for older adults with worry symptoms and co-occurring cognitive dysfunction. International Journal of Geriatric Psychiatry, 29, 991-1000. doi:10.1002/gps.4086

Lykins, E. B., Baer, R., \& Gottlob, L. R. (2012). Performance based tests of attention and memory in long-term mindfulness meditators and demographically matched nonmediators. Cognitive Therapy Research, 36, 103-114. doi:10.1007/s10608010-9318-y

Mallya, S., \& Fiocco, A. J. (2016). Effects of mindfulness training on cognition and well-being in healthy older adults. Mindfulness, 7, 453-465. doi:10.1007/s12671015-0468-6

Meyer, J. S., \& Novak, M. A. (2012). Minireview: Hair cortisol: A novel biomarker of hypothalamic-pituitary-adrenocortical activity. Endocrinology, 153, 4120-4127. doi:10.1210/en.2012-1226

Moynihan, J. A., Chapman, B. P., Klorman, R., Krasner, M. S., Duberstein, P. R., Brown, K. W., \& Talbot, N. L. (2013). Mindfulness-based stress reduction for older adults: Effects on executive function, frontal alpha asymmetry and immune function. Neuropsychobiology, 68, 34-43. doi:10.1159/000350949

Muiños, M., \& Ballesteros, S. (2015). Sports can protect dynamic visual acuity from aging: A study with young and older judo and karate martial arts athletes. Attention, Perception \& Psychophysics, 77, 2061-2073. doi:10.3758/s13414-015-0901-x

Osterberg, L., \& Blaschke, T. (2005). Adherence to medication. The New England Journal of Medicine, 353, 487-497. doi:10.1056/NEJMra050100

Oswald, W. D., \& Roth, E. (1987). Der Zahlen-Verbindungs-Test (ZVT). Göttingen, Germany: Hogrefe Verlag fuer Psychologie. 
Peters, M., Laeng, B., Latham, K., Jackson, M., Zaiyouna, R., \& Richardson, C. (1995). A redrawn Vandenberg and Kuse Mental Rotations Test: Different versions and factors that affect performance. Brain and Cognition, 28, 39-58.

Petermann, F. (2012). Wechsler Adult Intelligence Scale-Fourth Edition. Deutschsprachige Adaptation nach David Wechsler. Frankfurt, Germany: Pearson.

Piazza, J. R., Charles, S. T., Stawski, R. S., \& Almeida, D. M. (2013). Age and the association between negative affective states and diurnal cortisol. Psychology and Aging, 28, 47-56. doi:10.1037/a0029983

Pietsch, S., \& Jansen, P. (2012). Different mental rotation performance in students of music, sports and education science. Learning and Individual Differences, 22, 159-163. doi:10.1016/j.lindif.2011.11.012

Russell, E., Koren, G., Rieder, M., \& Van Uum, S. (2012). Hair cortisol as a biological marker of chronic stress: Current status, future directions and unanswered questions. Psychoneuroendocrinology, 37, 589-601. doi:10.1016/j.psyneuen. 2011.09.009

Scheier, M. F., Carver, C. S., \& Bridges, M. W. (1994). Distinguishing optimism from neuroticism (and trait anxiety, self-mastery, and self-esteem): A reevaluation of the Life Orientation Test. Journal of Personality and Social Psychology, 67, 1063-1078. doi:10.1037/0022-3514.67.6.1063

Schlotz, W., Kumsta, R., Layes, I., Entringer, S., Jones, A., \& Wüst, S. (2008). Covariance between psychological and endocrine responses to pharmacological challenge and psychosocial stress: A question of timing. Psychosomatic Medicine, 70, 7, 787-796.

Schulz, P., \& Schlotz, W. (1999). Trierer Inventar zur Erfassung von chronischem Streß (TICS): Skalenkonstruktion, teststatistische Überprüfung und Validierung der Skala Arbeitsüberlastung. Diagnostica, 45, 8-19. doi:10.1026//0012-1924. 45.1 .8

Schulz, P., Schlotz, W., \& Becker, P. (2004). Trierer Inventar zum chronischen stress. Göttingen, Germany: Hogrefe.

Shepard, R. N., \& Metzler, J. (1971). Mental rotation of three-dimensional objects. Science, 701-703.

Singh-Manoux, A., Kivimaki, M., Glymour, M. M., Elbaz, A., Berr, C., Ebmeier, K. P., ... Dugravot, A. (2012). Timing of onset of cognitive decline: Results from Whitehall II prospective cohort study. British Medical Journal (Clinical Research Ed.), 344, d7622. doi:10.1136/bmj.d7622

Stalder, T., \& Kirschbaum, C. (2012). Analysis of cortisol in hair-state of the art and future directions. Brain, Behavior, and Immunity, 26, 1019-1029. doi:10.1016/j. bbi.2012.02.002

Staufenbiel, S. M., Penninx, B. W., Spijker, A. T., Elzinga, B. M., \& van Rossum, E. F. (2013). Hair cortisol, stress exposure, and mental health in humans: 
A systematic review. Psychoneuroendocrinology, 38, 1220-1235. doi:10.1016/ j.psyneuen.2012.11.015

Strawbridge, W. J., Deleger, S., Roberts, R. E., \& Kaplan, G. A. (2002). Physical activity reduces the risk of subsequent depression for older adults. American Journal of Epidemiology, 156, 328-334. doi:10.1093/aje/kwf047

Steyer, R., Schwenkmezger, P., Notz, P., \& Eid, M. (1997). Der Mehrdimensionale Befindlichkeitsfragebogen MDBF [Multidimensional Mood Questionnaire]. Göttingen, Germany: Hogrefe.

Tartaglia, F. (2003). Shotokan-Kata bis zum Schwarzgurt (Band 1). Göttingen, Germany: Spectra-Verlag.

Tsai, A. C., Chi, S.-H., \& Wang, J.-Y. (2015). Association of perceived stress with depressive symptoms in older Taiwanese: Results of a population-based study. Geriatrics \& Gerontology International, 15, 535-543. doi:10.1111/ggi.12307

Vernon, P. A. (1993). Der Zahlen-Verbindungs-Test and other trail-making correlates of general intelligence. Personality and Individual Differences, 14, 35-40.

Virtanen, M., Ferrie, J. E., Batty, G. D., Elovainio, M., Jokela, M., Vahtera, J., ... Kivimäki, M. (2015). Socioeconomic and psychosocial adversity in midlife and depressive symptoms post retirement: A 21-year follow-up of the Whitehall II study. The American Journal of Geriatric Psychiatry: Official Journal of the American Association for Geriatric Psychiatry, 23, 99.e1-109.e1. doi:10.1016/j. jagp.2014.04.001

Ware, J., Kosinski, M., \& Keller, S. D. (1996). A 12-Item Short-Form Health Survey: Construction of scales and preliminary tests of reliability and validity. Medical Care, 34, 220-233. doi:10.1097/00005650-199603000-00003

Young, J., Angevaren, M., Rusted, J., \& Tabet, N. (2015). Aerobic exercise to improve cognitive function in older people without known cognitive impairment. The Cochrane Database of Systematic Reviews, 4, 1-141. CD005381. doi:10. 1002/14651858.CD005381.pub4

Zigmond, A. S., \& Snaith, R. P. (1983). The Hospital Anxiety and Depression Scale. Acta Psychiatrica Scandinavica, 67, 361-370. doi:10.1111/j.1600-0447.1983. tb09716.x

Zoogman, S., Goldberg, S. B., Hoyt, W. T., \& Miller, L. (2015). Mindfulness interventions with youth: A meta-analysis. Mindfulness, 6, 290-302. doi:10.1007/ s12671-013-0260-4

\section{Author Biographies}

Petra Jansen is a professor in the Institute of Sport Science, University of Regensburg, Germany. Her main research interests include the topics of the influence of 
motor behavior and mindfulness on cognition and emotion, cognitive development, and gender differences in cognition.

Katharina Dahmen-Zimmer is an assistant professor in the Institute of Psychology, University of Regensburg, Germany. Her research interests include mobility and driving behavior of elderly people, effects of exercise on cognition, emotion and self-efficacy of younger and older adults.

Brigitte M. Kudielka is a professor in the Institute of Psychology, University of Regensburg, Germany. Her main research interests are psychological and biological determinants of individual stress regulation. Main aims are to identify mechanisms explaining how stress-related health disturbances develop and to identify psychological and biological factors of inter- and intraindividual stress vulnerability.

Anja Schulz is a PhD student at the Technische Universität Dresden, Germany, and working as clinical psychologist at the Clinic for Forensic Psychology and Psychotherapy, Parsberg, Germany. Her main research interests include psychological correlates of deviant sexual behavior against children and the etiology of as well as attention-based mechanisms associated with sexual interest in children. 